\title{
Partidos políticos y democracia participativa: estatus político y perfil asociativo en los presupuestos participativos de Brasil $^{1}$
}

Political parties and participatory democracy: political status and associative profile in participatory budgeting of Brazil

\author{
WAGNER ROMÃO \\ Universidade Estadual de Campinas, Brasil
}

\section{Cómo citar/Citation}

Romão, W. (2016). Partidos políticos y democracia participativa: estatus político y perfil asociativo en los presupuestos participativos de Brasil. Revista Española de Ciencia Política, 42, 69-97. Doi: http://dx.doi.org/10.21308/ recp.42.03

\section{Resumen}

El artículo explora las relaciones entre el estatus politico de las estructuras del presupuesto participativo (PP) y el perfil asociativo de los representantes de la sociedad civil en cuatro experiencias brasileñas. Nuestro foco es considerar la ubicación de la coordinación del PP en el gobierno municipal, las características de su consejo y la afiliación partidaria de los consejeros de la sociedad civil. Los resultados muestran que cuanto más alto es el estatus político del PP en la Administración Local, el consejo es más disputado y ocupado por representantes afiliados al partido político en el poder. Es decir, se produce un conflicto donde los grupos más organizados dirigen sus estrategias de ocupación del espacio político hacia el PP.

Palabras clave: presupuesto participativo, partidos políticos, participación, gobierno local, sociedad civil, Brasil.

\section{Abstract}

The article explores the relationships between the political status of coordinating structures of participatory budgeting (PB) and the associative profile of civil society representatives in four

1. Este articulo fue concebido en el ámbito de la investigación de postdoctorado del autor en el Centro de Estudios de la Metrópoli, vinculado al Centro Brasileño de Análisis y Planeamiento (CEM-CEBRAP) 
Brazilian experiences. Our focus is to take into consideration the location of the coordination office of PB within the municipal government, the characteristics of the PB Council and the party affiliation of civil society councilors. The results show that the higher political status of $\mathrm{PB}$ at local government the more the council will be fought over and occupied by representatives affiliated to the incumbent political party. That is to say, a conflict occurs where the most organized groups channel their strategies to occupy the political space towards the PB.

Keywords: participatory budgeting, political parties, participation, local government, civil society, Brazil.

\section{INTRODUCCIÓN}

Por muchos años, teóricos y ciudadanos mantuvieron la esperanza de que la democracia participativa podría ser la solución para la crisis de representación del sistema político, que es también la crisis de los partidos políticos. Pero, ¿qué ocurre si descubrimos que puede haber una relación positiva entre el éxito de experiencias de democracia participativa -como el presupuesto participativo (PP) - y la mayor presencia en las mismas de ciudadanos afiliados a los partidos?

En este artículo presentamos un estudio sobre las experiencias de PP con el foco puesto en su interacción con las estructuras administrativas de los gobiernos locales y en el perfil de la sociedad civil movilizada en el proceso participativo. Para ello, nos basamos en una investigación empírica hecha con base en cuatro casos de Brasil, en municipios de la región metropolitana de São Paulo.

Trabajos como el de Navarro (2003), Baiocchi (2005), Wampler (2007), Avritzer (2009), Baiocchi y Heller (2009), Romão (2011), Baiocchi, Heller y Silva (2011), Leubolt et al. (2012), Montambeault (2012), Ganuza y Baiocchi (2013) y otros autores han hecho hincapié en un análisis que considera las dificultades, desafíos y límites de las experiencias participativas en su relación con las estructuras de la democracia tradicional representativa, es decir, con el sistema político, los partidos políticos, los procesos electorales y la burocracia en las administraciones locales. Es este el marco analítico en el que se inserta este trabajo, que intenta avanzar en el debate sobre las relaciones entre el estatus político del PP en el gobierno y el perfil de la sociedad civil movilizada por el proceso participativo.

Desde una metodología de análisis basada en el estudio de la organización de las secretarías en las alcaldías, de la estructura de participación de la sociedad civil y su perfil asociativo, tratamos en primer lugar de marcar las diferencias institucionales de las propuestas de PP, lo que nos permitirá establecer cómo las mismas llevan a resultados diferentes cuando hablamos de la estructura del proceso participativo. Pero más allá de eso -y entendemos que esta es la principal contribución de este trabajo a la literatura científica-, también se indica cómo estas diferencias pueden afectar al perfil de la sociedad civil que participa, especialmente en la presencia de ciudadanos afiliados a los partidos políticos. 
Así, nuestra principal hipótesis es que, incluso experiencias de diseño institucional muy semejantes -como son las de Brasil- pueden contener variaciones que producen efectos en la capacidad del PP de incidir en decisiones en cuanto al presupuesto y en cuanto a la sociedad civil movilizada por este. Esto se hace visible a través de tres factores: a) el estatus político del PP en el seno del gobierno local, caracterizado más claramente en este artículo por la ubicación de la estructura de gobernanza del PP en la administración de la municipalidad; b) los cambios o permanencias en su diseño institucional, caracterizado más claramente por la composición (gobierno/sociedad civil) del consejo del PP o de estructuras adjuntas, y c) el perfil asociativo de la representación de la sociedad civil en el consejo del PP.

En este estudio encontramos una correlación fuerte entre la ubicación de la estructura del PP en los órganos de planificación de las alcaldías; la división paritaria entre miembros del gobierno y de la sociedad civil en el consejo del PP, y la participación de personas afiliadas a los partidos políticos en el consejo del PP. Más allá de esta correlación, defendemos que el estatus político del PP en las alcaldías, y su diseño institucional son determinantes para la conformación del perfil de la sociedad civil que participa en el PP, especialmente en lo que se refiere a la densidad de la participación o no de personas afiliadas a los partidos políticos en la estructura de participación.

Después de esta breve introducción, presentamos ejemplos de cómo la literatura ha tratado el problema de la "partidarización» de la sociedad civil movilizada por los presupuestos participativos. A continuación ofrecemos algunas consideraciones metodológicas y preliminares de los casos. Seguidamente se presenta cada caso en conformidad con lo propuesto anteriormente y, por último, se ofrecen las conclusiones del artículo.

\section{SOBRE LOS PRESUPUESTOS PARTICIPATIVOS Y EL ESTADO DE LA CUESTIÓN}

Los presupuestos participativos son iniciativas gubernamentales de participación de los ciudadanos, no elegidos en procesos electorales tradicionales, en la toma de decisiones sobre los presupuestos, que nacieron en Brasil en alcaldías lideradas por el Partido de los Trabajadores a finales de los años ochenta y comienzos de los noventa del pasado siglo. El caso más célebre es Porto Alegre, cuyo método de participación le generó reconocimiento mundial y a partir del cual se transmitió hacia ciudades de los cinco continentes (Santos, 1998; Porto de Oliveira, 2016).

Es verdad que, desde la matriz portoalegrense, la práctica del PP tiene muchas diferencias, justificables fundamentalmente por las distintas formas de «aterrizaje» del modelo en estructuras de gobierno también muy distintas en el mundo. Por esto, Sintomer et al. (2013) proponen una definición mínima de los PP en la que debe cumplirse que a) la dimensión financiero presupuestaria debe discutirse; b) que dicha discusión abarque el nivel municipal o por lo menos un distrito; c) el proceso debe ser 
recurrente o continuo; d) debe haber algún foro de deliberación pública en foros específicos, y e) debe haber algún grado de rendición de cuentas de los resultados.

Desde el clásico caso de Porto Alegre, las estructuras locales del presupuesto participativo en Brasil tienen características similares: son procedimientos de participación abiertas a los ciudadanos, que empiezan con sesiones plenarias organizadas a partir de una base territorial, en los barrios o distritos de las ciudades. En estos plenarios se eligen y se priorizan propuestas de acción en servicios públicos y también son elegidos los consejeros responsables de llevar las demandas priorizadas a la más alta esfera de deliberación, el consejo del PP. En el consejo, formado solo por miembros de la sociedad civil o en conjunto con representantes del gobierno local, se elabora la propuesta de presupuesto que va al Ayuntamiento para su aprobación final. Este modelo «ideal» tiene variaciones que impactan, sobre todo, en la autonomía de decisión del consejo del PP.

La preocupación de este estudio es identificar las posibles relaciones entre el estatus político del PP en el contexto del gobierno local y las características de la sociedad civil movilizada. Además del estudio de la estructura del poder del gobierno local, decidimos centrar nuestra atención en el consejo del PP en los cuatro casos por ser el espacio de más poder en el contexto del proceso de participación, donde se encuentran los consejeros que representan a las regiones de la ciudad.

El interés en el perfil de los ciudadanos movilizados por las experiencias del PP está relativamente presente en la literatura $y$, en algunos estudios, pasó directamente a la cuestión de la afiliación a los partidos políticos. Bajo el signo de la polaridad cooptación/ autonomía, Navarro (2003) señala cómo, en el caso de Porto Alegre, un clientelismo tradicional habría dado lugar a un clientelismo de nuevo tipo - «partidario»- donde las principales decisiones obedecían a la lógica de las juntas entre los operadores partidarios-gubernamentales con los líderes de las comunidades. Esta lógica habría sido fundamental para la declaración del PP como un instrumento del enfrentamiento entre el Partido de los Trabajadores (PT) y el Partido Democrático del Trabajo (PDT) en el marco del PP y de las disputas por las asociaciones comunitarias.

Baiocchi (2005), con su estudio etnográfico en Porto Alegre, ha encontrado rasgos distintivos del PP entre las regiones, relacionados con los orígenes de las actividades comunitarias y respeto de la relación que la sociedad civil local tuvo con los sucesivos Gobiernos y partidos políticos, especialmente PDT y PT. Wampler (2004), en una encuesta aleatoria telefonica realizada en el año 2003 con una muestra de delegados del PP de São Paulo, identificó una tasa del 37 \% de los entrevistados que afirmaron estar afiliados a partidos políticos y, entre ellos, el $87 \%$ dijo pertenecer a partidos de izquierda -PT, Partido Socialista Brasileño (PSB), Partido Comunista de Brasil (PCB) y Partido Verde (PV)-. Nylen (2002), en su investigación con delegados del PP en 1998, obtuvo un resultado del 23,5\% de afiliados a partidos en Belo Horizonte y del $39,3 \%$ en Betim. En el caso de los pequeños municipios, como Icapuí (Ceará), de 16000 habitantes, Teixeira (2003) encontró que el 63,9\% de los consejeros del PP eran funcionários públicos y el 8,3\% eran proveedores de servicios para el ayuntamiento.

Montambeault (2011) -también en el marco de la polaridad cooptación/autonomía- ha comparado los casos de Porto Alegre, Belo Horizonte y Recife con el fin de 
establecer la manera en que las prácticas informales -es decir, que ocurren al margen de las reglas formales y del diseño institucional de las experiencias- potencian el "aprendizaje cívico y democrático». Aparte de las similitudes de los diseños institucionales en los casos, hay diferencias relativas a las estrategias de control del proceso participativo, sea por actores políticos en el gobierno o en los partidos, o por líderes de asociaciones comunitarias. La investigación demuestra que en Porto Alegre y en Belo Horizonte la relación de los partidos se realizaba a través de los canales formales de los presupuestos participativos, pero en Recife no ocurría así, e incluso algunos delegados entrevistados «se veían a sí mismos como trabajadores del gobierno local en las comunidades y no como representantes de su comunidad». Para nuestro propósito interesa destacar que Montambeault ha descubierto que en Recife, como afirmaba un asesor del PP, «los foros están informalmente ocupados por partidos, son utilizados para luchas políticas al igual que los demás espacios públicos. El PT tiene una mayoría, pero los otros partidos organizan también a los líderes locales en los barrios donde tienen sus bases electorales y militantes» (2011: 158). Esto también ocurre en casos de éxito como Porto Alegre (Filomena, 2006).

Pero lo que nos interesa aquí no es solo identificar la presencia de afiliados en los partidos políticos en el consejo del PP. Este elemento se convirtió en un factor importante a partir de los resultados de las investigaciones llevadas a cabo desde que fue posible identificar una relación directa entre el estatus político más alto del PP dentro de la administración municipal y una mayor presencia de afiliados a los partidos en los consejos del PP. Sin embargo, el fenómeno de la multiafiliación es bastante frecuente en estas instancias de participación y en la acción colectiva en general (Mische, 2009). Así, tratamos de desarrollar una investigación donde se articulen otras afiliaciones institucionales de los consejeros. Y, como veremos, estas tienen sentido en su relación con la conformación del PP en cada caso.

Como instrumental de análisis, utilizamos la metodología de redes sociales (Hanneman, 2001), con el fin de producir un retrato del perfil de la sociedad civil local en el consejo del PP. Esta metodología nos permite visualizar las múltiples filiaciones institucionales de los consejeros, para hacer un análisis del perfil de la sociedad civil que llega al consejo del PP y poder compararlo con otros perfiles distintos en otras ciudades. Así, presentamos los vínculos que unen a los consejeros del PP con los movimientos sociales, organizaciones de la sociedad civil, sindicatos, grupos y partidos políticos, para entender cómo -a través de estos agentes sociopolíticos- suele variar, en cada caso estudiado, el público movilizado para la participación política y la representación de los intereses en el nivel más alto del presupuesto participativo. Intentamos, de esa manera, comprobar qué parte de la sociedad civil es, de hecho, activada por las experiencias del PP y, con la mirada puesta en su heterogeneidad, relacionar en cada una de las experiencias estudiadas el perfil de la sociedad civil participante en el consejo del PP, tanto con su estatus político como con el diseño de dicho consejo. Esto nos permitirá demostrar cómo los representantes de la sociedad civil reaccionan de manera distinta a las distintas propuestas institucionales del PP. 
Por último, y con el fin de que nuestra perspectiva ofrezca una contribución teórica relevante para los estudios de PP en el mundo, optamos por relacionar nuestros casos con los modelos conceptuales de participación ciudadana de Sintomer et al. (2013). Estos autores sugieren tipos ideales de experiencias de PP a partir del análisis de casos en los cinco continentes, articulando las formas en que se combinan la estructura de acción participativa de los gobiernos locales y el modo en que se le permite a la sociedad civil local intervenir de manera más o menos participativa. En concreto, esta tipología identifica seis modelos de participación, con la posibilidad de que los casos migren de un modelo a otro. Aunque no podemos realizar una larga exposición, a continuación ofrecemos un resumen de cada uno de ellos:

1. Democracia participativa. En este modelo, la sociedad civil participa en la toma de decisiones de un modo efectivo. Los participantes forman parte de grupos subalternos de la ciudad. Es la materialización de las ideas de «democracia directa» $\mathrm{y}$ «justicia social».

2. Democracia de proximidad. Aquí, la sociedad civil tiene poca autonomía. El gobierno hace una "escucha selectiva» de una sociedad civil escogida. No se trata de un instrumento de justicia social y se refuerza el marco del gobierno representativo.

3. Modernización participativa. Este modelo pone el énfasis en las nuevas estrategias de gestión, con poco protagonismo de la sociedad civil y con el predominio de la idea de un «buen gobierno», que aumenta la legitimidad de las políticas públicas. La idea de justicia social queda bastante relegada.

4. Participación de los stakeholders. El protagonismo lo asumen las empresas privadas y los gobiernos locales. Los ciudadanos son socios minoritarios en la participación.

5. Neocorporativismo. La consulta se realiza con los diversos actores que se consideran importantes desde un punto de vista representativo: organizaciones no gubernamentales $(\mathrm{ONG})$, sindicatos, grupos sociales organizados, líderes e instituciones locales. La participación es coordinada por el gobierno local, en la búsqueda de un consenso social obtenido mediante la mediación de intereses, valores y demandas de los diversos segmentos de la sociedad.

6. Desarrollo comunitario. La participación vuelve a la fase de implementación de un proyecto. En este modelo los procedimientos son claros y la deliberación se caracteriza por su alta calidad. El rol de intermediación de las ONG es decisivo, poniéndose el énfasis en la inclusión de organizaciones comunitarias.

Para los fines de nuestro estudio sobre los casos de Brasil, decidimos utilizar de manera conjunta los modelos de «democracia participativa» $\mathrm{y}$ «modernización participativa», y mantener por separado los modelos de "democracia de proximidad" $\mathrm{y}$ «neocorporativismo». Por otra parte, los modelos de "participación de los stake-holders» y de «desarrollo comunitario» están muy poco presentes en Brasil. Considerando estos modelos, es posible afirmar que en Brasil se conectan las ideas de democracia partici- 
pativa y modernización participativa. Las experiencias que han tenido más éxito conectan la idea de participación autónoma de la sociedad civil con la idea de modernización administrativa, especialmente en la definición de reglas más claras de transferencia de recursos públicos a las regiones de la ciudad, vinculada a las pretensiones de mejorar la justicia social. Por lo tanto, mantendremos juntos estos dos modelos en nuestro análisis. Las experiencias de São Bernardo do Campo y, en parte, de Guarulhos, se encuadran en este modelo.

La diferencia fundamental entre la democracia de proximidad y el neocorporativismo -tal como es presentada por los autores citados- es la fuerza y la autonomía de la representación de la sociedad civil, que es frágil en el primer modelo y fuerte en el segundo; es decir, la diferencia entre una sociedad civil más o menos invertebrada -o lo que es lo mismo, una sociedad civil con menos instituciones en los foros y consejosy otra donde interesa la representación institucional de sindicatos, ONG, iglesias, etc. En algunos de nuestros casos, la trayectoria del PP sugiere un equilibrio, con momentos de interlocución más general con la sociedad -y por esto más desigual en términos de autonomía- y otros de una interlocución más focalizada hacia sectores institucionales más específicos, y por ende, donde la autonomía es más fuerte. Este equilibrio se observa muy claramente en los casos de Diadema y Guarulhos. Por otra parte, en el caso de Osasco percibimos una fuerte semejanza con el modelo de democracia de proximidad, al ser una experiencia poco estructurada internamente dentro de la alcaldía y progresivamente con poca autonomía de la sociedad civil.

En las conclusiones del artículo volveremos a los modelos, lo que nos ayudará a hacer una evaluación del sentido político de los casos y una reflexión sobre la presencia de afiliados de partidos políticos en ellos. Previamente ofrecemos una explicación detallada de la metodología y del análisis empírico de los casos de las municipalidades.

\section{METODOLOGÍA Y PRESENTACIÓN DE LOS CASOS}

La siguiente tabla señala algunas diferencias relevantes entre las experiencias de presupuesto participativo en Diadema, Osasco, São Bernardo do Campo y Guarulhos. La elección de los casos se explica por varias razones. En primer lugar, los cuatro son grandes municipios de la región metropolitana de São Paulo. De esta forma, se trató de mantener constante que los municipios estudiados constituyen partes del principal centro urbano del país, lo que define en gran medida las características de la sociabilidad de estos municipios, así como lo que algunos llamarían su «tradición asociativa». Las cuatro ciudades tienen una historia relevante de acción colectiva y de actividad de movimientos sociales y sindicales; son ciudades con una dinámica social eminentemente urbana, industrial y metropolitana; un rasgo fundamental de su estructura urbana es la pronunciada dicotomía centro/periferia, es decir, son lugares donde existe un alto grado de desigualdad social, con centros muy urbanizados y periferias con enormes dificultades en términos de urbanización, vivienda, etc. 
TABLA 1.

Características del presupuesto participativo en Diadema, Osasco, São Bernardo do CAMPo y Guarulhos (2012)

\begin{tabular}{|c|c|c|c|c|}
\hline & DIADEMA & OSASCO & $\begin{array}{c}\text { SÃO } \\
\text { BERNARDO } \\
\text { DO CAMPO }\end{array}$ & GUARULHOS \\
\hline $\begin{array}{l}\text { Inicio de las } \\
\text { actividades }\end{array}$ & 2001 & 2005 & 2009 & 2001 \\
\hline $\begin{array}{l}\text { Composición } \\
\text { actual del Consejo }\end{array}$ & $\begin{array}{l}\text { Sociedad civil (35), } \\
\text { gobierno (20) }\end{array}$ & $\begin{array}{c}\text { Solamente } \\
\text { sociedad civil } \\
(46)\end{array}$ & $\begin{array}{l}\text { Sociedad civil } \\
\text { (20), gobierno } \\
(20)\end{array}$ & $\begin{array}{l}\text { Sociedad civil (44), } \\
\text { gobierno (30) }\end{array}$ \\
\hline $\begin{array}{l}\text { Periodicidad de } \\
\text { los encuentros del } \\
\text { Consejo }\end{array}$ & Media (3 meses) & $\begin{array}{l}\text { Baja-esporádica } \\
\text { (6 meses) }\end{array}$ & $\begin{array}{l}\text { Alta }(2-4 \\
\text { semanas })\end{array}$ & Media (2 meses) \\
\hline $\begin{array}{l}\text { Ubicación del PP } \\
\text { en el gobierno }\end{array}$ & $\begin{array}{l}\text { Coordinación del } \\
\text { PP por la } \\
\text { Coordinación de } \\
\text { Participación } \\
\text { Popular y por el } \\
\text { gabinete del alcalde }\end{array}$ & $\begin{array}{l}\text { Coordinación del } \\
\text { PP por el } \\
\text { gabinete del } \\
\text { alcalde }\end{array}$ & $\begin{array}{l}\text { Departamento de } \\
\text { Planificación } \\
\text { Participativa, y } \\
\text { por la Secretaría } \\
\text { de Presupuesto y } \\
\text { Planificación } \\
\text { Participativa } \\
\text { (SPPP) }\end{array}$ & $\begin{array}{l}\text { Coordinación del } \\
\text { PP por la } \\
\text { Secretaría de } \\
\text { gobierno }\end{array}$ \\
\hline División regional & 13 regiones & 19 regiones & 20 regiones & 22 regiones \\
\hline Población (2010) & 395.333 & 714.950 & 803.906 & 1.221 .979 \\
\hline $\begin{array}{l}\text { Nombre del } \\
\text { Consejo }\end{array}$ & $\begin{array}{l}\text { Consejo del } \\
\text { Presupuesto } \\
\text { Participativo } \\
\end{array}$ & $\begin{array}{l}\text { Consejo del } \\
\text { Presupuesto } \\
\text { Participativo }\end{array}$ & $\begin{array}{c}\text { Consejo } \\
\text { Municipal del } \\
\text { Presupuesto }\end{array}$ & $\begin{array}{l}\text { Consejo del } \\
\text { Presupuesto } \\
\text { Participativo }\end{array}$ \\
\hline
\end{tabular}

Fuente: elaboración propia.

Otra variable presente en las cuatro experiencias es la coincidencia del partido político del alcalde en el periodo de trabajo de campo de la investigación (2011-2012). Los cuatro municipios estaban entonces gestionados por el Partido de los Trabajadores (PT). Como veremos, sin embargo, tienen modelos muy distintos de gestión del $\mathrm{PP}$, lo que indica que aunque el PT haya sido el partido político brasileño que ha puesto en marcha más experiencias de PP en los gobiernos municipales, estas experiencias presentan distintos grados de estatus político en sus gobiernos.

Mantenidas constantes las variables antes señaladas -el tamaño de la población, las características urbanas generales de los municipios y el partido político del alcalde- se ha considerado importante incluir la variación en la longevidad de las experiencias. Así, tenemos dos experiencias que se iniciaron en 2001 (Diadema y Guarulhos), una en 2005 (Osasco) y otra en 2009 (São Bernardo do Campo). Este factor parece ser muy relevante si se establecen los cambios en la gestión del proceso participativo como un componente de la investigación sobre los cambios de su estatus político. Las experiencias de PP pueden variar con mayor o menor grado en sus procesos internos, en su 
intento de ampliación o cambio del público al que se incorpora a la participación - esto se observa claramente en el caso de la experiencia de Diadema- o en su intento de armonizar la relación del PP con la estructura del gobierno. En este último caso, puede darse incluso la irrelevancia del PP, como ocurre en la experiencia de Osasco.

MAPA 1.

Región Metropolitana de São Paulo

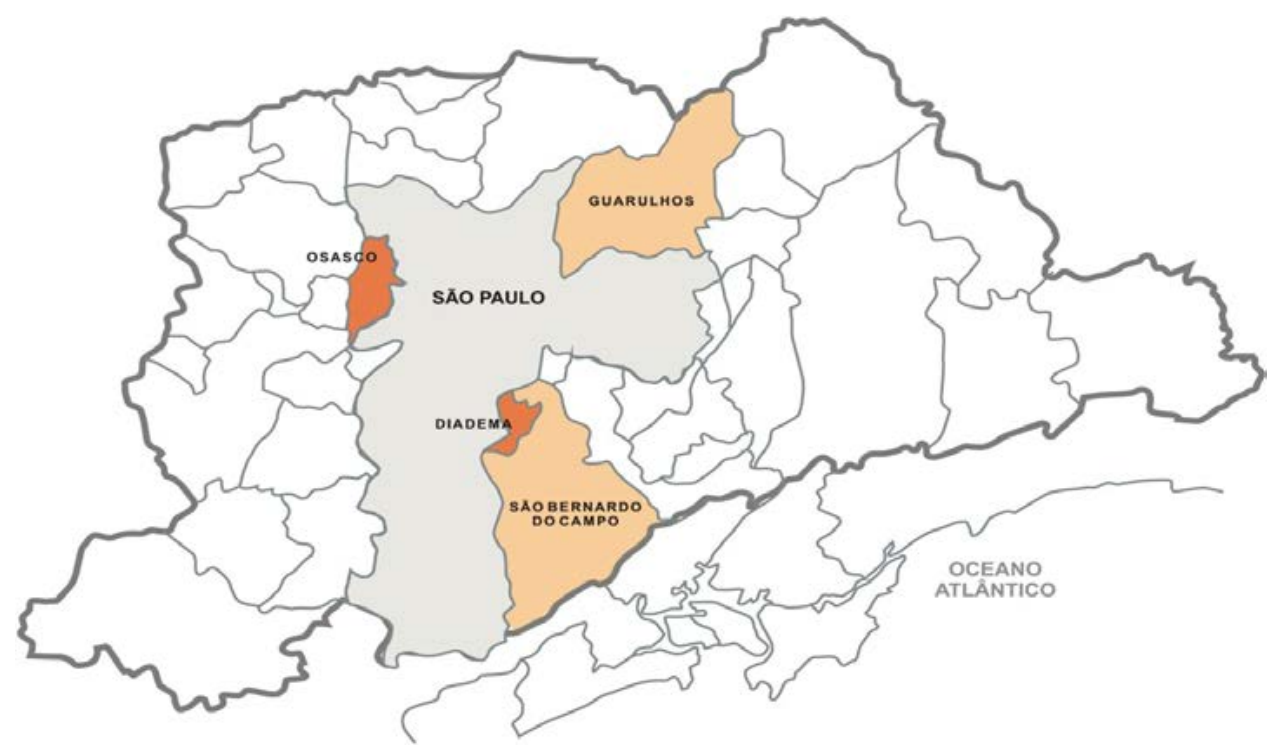

Fuente: elaboración propia.

Por lo demás, en todos los casos también se repite la siguiente situación política en relación con el presupuesto participativo y su estatus en el gobierno: en general, al comienzo de los mandatos -o en el primero de ellos- existe la necesidad política de aportar símbolos de cambio frente a los gobiernos anteriores. Y, como elemento simbólico, fomentar la participación directa de los ciudadanos en los asuntos públicos a través del PP puede ser algo bastante importante, y se convierte además en una especie de mensaje a los miembros del partido y a los partidarios más cercanos de que el nuevo gobierno está cumpliendo con las promesas de democratización y de cambio en la cultura política local. Al menos hasta el 2012, el PP y el fomento de la participación directa fueron percibidos como una marca de distinción de los gobiernos del PT o de izquierda. Sin embargo, en general con el paso de los años -y con mayor o menor impacto en la dinámica de participación- se ha producido una naturalización del PP. Este fenómeno también ha afectado al perfil político-institucional de los participantes más activos del PP, los consejeros del presupuesto participativo.

El período de este estudio comprende desde finales de 2011 a finales de 2012. El trabajo de campo consistió en la observación participante de las sesiones y reuniones 
de los consejos de los presupuestos participativos en los cuatro municipios. También se realizaron entrevistas con los coordinadores responsables de PP y ocasionalmente con funcionarios de sus equipos. Estas entrevistas tuvieron dos objetivos principales: a) reconstruir la trayectoria del presupuesto participativo en la ciudad, y b) entender el papel del PP en el contexto del gobierno municipal. La información obtenida por estas vías fue complementada con el análisis de documentos, incluyendo reglamentos, material de publicidad y otras publicaciones.

Hubo un trabajo de investigación específico con los consejeros del presupuesto participativo, es decir, con los representantes de la sociedad civil. En las reuniones del consejo de PP se hizo un cuestionario a los consejeros sobre su perfil político-institucional y socioeconómico, así como un conjunto de preguntas sobre el funcionamiento del PP. Una parte de los consejeros no estuvo presente en las reuniones en la fecha de la aplicación del cuestionario, por lo que tuvimos que desarrollar otra estrategia para obtener información sobre ellos. Para ello, escogimos a cinco consejeros que, en una entrevista estructurada, nos proporcionaron información sobre el perfil político e institucional de los consejeros que faltaban; y en la medida en que la información proporcionada se repetía en las entrevistas, la dimos como verdadera. Por esta vía nos fue posible ampliar el número de miembros. Seguidamente, la información obtenida fue sistematizada y, con la ayuda del software UCINET, se desarrollaron sociogramas que proporcionan una visión clara de los vínculos asociativos de cada consejero con el fin de hacer inferencias acerca de la diversidad asociativa de los participantes del consejo en cada caso. Este procedimiento nos ha permitido establecer los perfiles de los consejeros e identificar sus vínculos con los diferentes actores institucionales (partidos políticos, asociaciones de vecinos, diferentes ONG, sindicatos, grupos de partidos políticos, campañas políticas, movimientos sociales, otros consejos, autoridades gubernamentales, etc.) con el fin de mostrar el grado de diversidad asociativa en cada una de las experiencias de PP.

\section{TRAYECTORIA DE LAS EXPERIENCIAS: ESTATUS POLÍTICO Y PERFIL ASOCIATIVO}

En este punto, describimos la trayectoria de las experiencias del PP, intentando discutir las razones políticas e institucionales relacionadas con cada experiencia. Pondremos el foco en la posición de la estructura organizativa del PP en el gobierno municipal, enfocado en el Consejo del PP.

\section{Diadema}

Aunque coordinadores del PP de Diadema afirman que en 1984 hubo una experiencia de PP en ese municipio -en el primer gobierno del PT en la ciudad- el ciclo 
del PP en Diadema comienza en 2001 con el regreso del PT al gobierno municipal, encabezado por José de Fillippi.

Diadema es un caso de longevidad del PP -la experiencia terminó solamente con la derrota del PT en las elecciones en 2012- ,lo que nos permite verificar los cambios constantes en sus características y en los intentos de ampliar la participación de los diferentes segmentos de la población de la ciudad. En 2001, el PP asume los mecanismos de participación utilizados en experiencias como la de Porto Alegre y Santo André: asambleas plenarias en barrios con elección de demandas y delegados/consejeros que ocuparán puestos en el consejo del PP. Otro mecanismo interno del PP, entre 2001 y 2004, fueran las asambleas temáticas centralizadas, por sectores como la educación, la salud, el saneamiento, el medio ambiente y el deporte. Entre 2005 y 2008 se eliminaron las asambleas temáticas y se produjo una expansión de las asambleas territoriales por los diferentes distritos de la ciudad.

El Consejo del PP entre 2001 y 2008 fue de representación paritaria entre el gobierno y la sociedad civil. Había dos representantes por distrito, hasta un total de 26 personas, sumado a 26 funcionarios del gobierno. Esto es un elemento importante porque creemos que cuanto mayor sea la representación del gobierno en el consejo, el compromiso con sus decisiones también será potencialmente mayor. Diadema pasa ocho años con una representación paritaria, pero en 2009, con el gobierno de Mario Reali, hay un cambio en la estructura del Consejo en el que el PP pasa de un modelo más cercano al de Santo André - con el PP conectado de una manera consistente con la elaboración del presupuesto- a un modelo en el que el PP se configura más como una «estrategia de movilización», es decir, de apoyo sociopolítico al gobierno o, como dicen Sintomer et al. (2013), de una democracia y modernización participativa hacia una democracia de proximidad. El Consejo pasa a estar formado, desde ese momento, por 80 personas, de los cuales 60 son representantes de la población y 20 funcionarios del gobierno. En 2011, debido a la escasa presencia de consejeros en las reuniones, que pasó a parecerse más a las asambleas, se disminuye el número de representantes a 35. En palabras del coordinador de la participación popular en una de las entrevistas: "Creemos que la composición del Consejo es un instrumento para fomentar la participación de los líderes, representantes de la ciudad. Así que si usted tiene un tablero más grande, usted tiene la oportunidad de tener más personas involucradas».

Es verdad que podría haber más diversidad de participantes en el consejo, pero la disminución de su incidencia sobre el presupuesto indica una disminución de su fuerza política. Este cambio en el número de representantes en el Consejo se corresponde con una nueva forma de división del presupuesto en el contexto del PP: el presupuesto asignado se dividió por zonas de la ciudad. Con un presupuesto municipal de cerca de 600 millones de reales, se dedicaron cinco al PP, es decir, solamente el 0,83\%, que a su vez ha de dividirse para los 13 distritos de la ciudad, teniendo en cuenta los ingresos de los hogares (se destinan más recursos para los distritos con menores ingresos). En sus mejores días, la cantidad dedicada al PP ascendía hasta el $3 \%$ del presupuesto. La situación de considerable ajuste presupuestario para el proceso participativo también produjo otro cambio que se hizo desde 2011 -pero que no se ha aplicado debido 
a la derrota electoral en 2012-: el PP debería afectar también a los gastos corrientes y no solo al gasto en inversiones.

En este contexto, la Administración propone la celebración del Congreso de la Ciudad. Es, por una parte, un mecanismo utilizado en muchas experiencias de PP -Porto Alegre, Santo André, Belo Horizonte y otros-, en el que se establece un proceso de planificación participativa, una alternativa al PP, con el objetivo de atraer a otras organizaciones de la sociedad civil y a otros grupos sociopolíticos. En Diadema, el Congreso se llevará a cabo con los Foros Regionales, con el fin de establecer contacto con un nuevo conjunto de grupos sociales, la mayor parte perteneciente a sectores de la clase media intelectual y ligada a movimientos sociales, sindicatos, iglesias y sectores empresariales. Por tanto, se plantea la participación institucional como método de captación de nuevos grupos sociopolíticos. También se trató de movilizar a los propios burócratas de la municipalidad, tal y como se destaca en una entrevista realizada a los coordinadores del PP. Es una estrategia, en palabras del coordinador de la participación popular de entonces, "para promover una relación cada vez más fuerte, cada vez más presente de la alcaldía en la ciudad, a través de los líderes de opinión».

El pequeño porcentaje del presupuesto para el PP (no más del $1 \%$ del total), la ampliación del número de participantes en el Consejo (alrededor de un tercio solo para el gobierno y dos tercios para la sociedad civil), y la creación de los llamados Foros Regionales, con invitación expresa a los líderes políticos de los distritos (como alternativa al formato abierto a cualquier ciudadano del PP), son las tres características que aumentan el papel del PP en Diadema, fundamentalmente como una estrategia de movilización sociopolítica donde la decisión sobre el presupuesto se deja en un segundo plano.

De hecho, la trayectoria del PP en Diadema se inicia muy cerca del modelo de democracia y modernización participativa, en el que el PP aparece como un instrumento de modernización y de conexión directa con la población en los distritos, en el centro de la acción de gobierno. Pero después, con la pérdida de recursos presupuestarios, la ampliación del Consejo del PP y la diferenciación en la estrategia de proximidad de la gestión con los liderazgos sociopolíticos de la ciudad en los Foros Regionales, pasa a predominar el modelo de democracia de proximidad y neocorporativismo. Hay, no obstante, una diferencia: en la estructura del Consejo se hace democracia de proximidad -baja autonomía, bajo poder de decisión, mucho más una estructura de conexión con sus propias bases políticas en la ciudad-, mientras que los Foros Regionales son una tentativa -en parte frustrada por la derrota en las elecciones de 2012- de establecer un contacto más directo "con quien importa» en la ciudad y de ampliar sus bases políticas.

El paso a un modelo mixto a caballo entre la democracia de proximidad y el neocorporativismo se refuerza por la ubicación de la Coordinación de Presupuesto Participativo en la estructura de gobierno, conectada a la Coordinación de Participación Popular, que a su vez formaba parte del gabinete del alcalde, que también albergaba a 
las siguientes coordinadoras: Políticas para las Mujeres, Políticas de Juventud para la Promoción de la Igualdad Racial y de los Consejos.

Figura 1.

Gestión del Presupuesto Participativo En Diadema

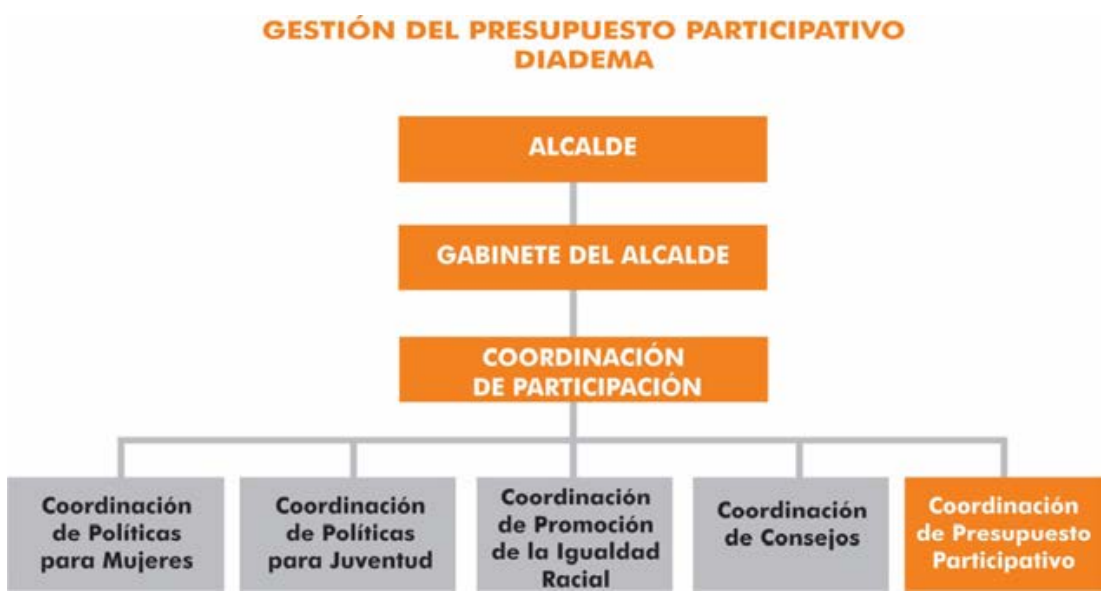

Fuente: elaboración propia basada en la investigación sobre el organigrama de la alcaldía de Diadema.

Por tanto, en el momento de la decisión sobre dónde instalar la estructura del PP, se optó por su conexión con una secretaría que dialogaba directamente con sectores con una serie de derechos específicos y que también servía de contacto entre los distintos consejos de políticas públicas. En este sentido, la participación tiene poca relación con la estructura de la toma de decisiones sobre el presupuesto o la planificación de la alcaldía, y está directamente relacionada con el diálogo con la sociedad y sus grupos específicos.

A partir de la metodología indicada anteriormente, construimos la red asociativa del Consejo del PP de Diadema. Es la red más grande y diversa de este estudio, y consta de 46 individuos. El PT es el nodo central de la red -19 consejeros están afiliados al PT-; pero también hay nodos periféricos, el más denso de los cuales es el movimiento de la vivienda, muy bien organizado en la ciudad y que utiliza el PP para la búsqueda de sus demandas territoriales. 
SOCIOGRAMA 1.

Diadema. Consejo del Presupuesto Participativo.

Representantes de la Sociedad Civil $(2012-2014)^{2}$

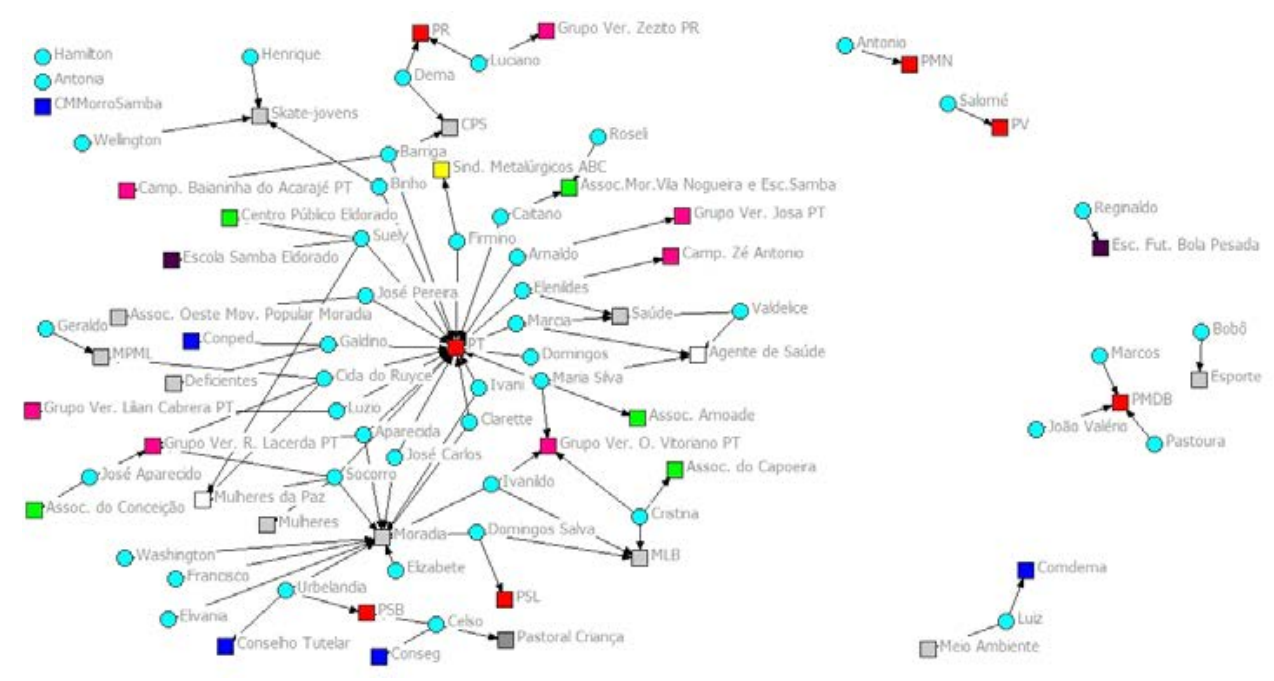

Fuente: Elaboración propia basada en las entrevistas.

Los vínculos de los individuos con los partidos políticos son importantes, sobre todo con el PT, pero también con partidos como el Partido del Movimiento Democrático Brasileño -PMDB- (3), PSB (2), Partido de la República -PR- (2), Partido Social Liberal -PSL- (1), PV (1) y Partido de la Movilización Nacional -PMN- (1). Además, la red en Diadema también presenta un considerable número de personas sin afiliación partidista y activistas de diferentes movimientos, como los jóvenes (patín), el deporte (fútbol), las mujeres, el Movimiento de Lucha en los Barrios (MLB), la salud y el movimiento por la vivienda, antes mencionado. También hay conexiones con las asociaciones de vecinos.

En comparación con los otros casos, Diadema tiene una menor proporción de personas del PT en la red y una mayor variedad de conexiones con movimientos sociales de variados matices. Y nos parece que ello es debido al hecho de que el PP en Diadema estuvo organizado más como un espacio de proximidad del gobierno del PT con los movimientos de su base sociopolítica y no tanto como un espacio de toma de decisiones, lo que atrajo al conjunto de los partidos políticos de la ciudad.

2. Para los sociogramas, los círculos azules se refieren a los consejeros(as); los cuadrados rojos a los partidos políticos; los cuadrados verdes a las asociaciones de vecinos; los cuadrados grises a los movimientos sociales; los cuadrados blancos a los órganos de gobierno; los cuadrados azules oscuro a los otros consejos gestores; los cuadrados negros a las ONG; los cuadrados amarillos a los sindicatos, y los cuadrados rosas a los grupos de los partidos políticos. 


\section{Guarulhos}

El PP empieza en Guarulhos en 1998, con la gestión de Jovino Candido, del PV. Candido reemplazó al exalcalde Nefi Tales, cuyo mandato fue revocado por corrupción. El PP fue uno de los componentes de las iniciativas de «saneamiento» del gobierno municipal y, por lo tanto, de modernización administrativa y de lucha contra la corrupción.

En 2001, con la elección del alcalde Eloi Pieta, del PT, el PP se convierte en una estrategia central del gobierno. La Coordinación del PP fue creada directamente vinculada al alcalde y la organización del PP fue semejante a la de Santo André (1997-2008).

El primer ciclo del PP en Guarulhos se llevó a cabo entre 2001 y 2002 e incluyó la elaboración y aprobación de los estatutos internos, la implementación de asambleas regionales y temáticas, la elección e investidura de los consejeros, las reuniones del Consejo del PP, el seguimiento de la ejecución del presupuesto y la evaluación del ciclo. La composición del Consejo de PP tenía la presencia de un representante de cada asamblea regional (16 en total) y uno de la asamblea temática cuyo objeto era el desarrollo económico y social. Además, la representación elegida en las asambleas tuvo la posibilidad de nombrar a los representantes de ocho organizaciones de la sociedad civil de la ciudad: dos de las sociedades de amigos del barrio y dos de los sindicatos -CUT y Fuerza Sindical, de la Orden de los Abogados del Brasil, de la Asociación Comercial e Industrial de Guarulhos, de la Federación de Industrias del Estado de São Paulo y de la Unión de Trabajadores de la Administración Pública Municipal de Guarulhos-. El Consejo se completó con 17 representantes del gobierno municipal, designados por el alcalde. Véase que el Consejo, en este momento inicial, se caracterizaba por combinar la representación regional con la representación «neocorporativista» de entidades de la sociedad civil.

En la segunda ronda se produjo un cambio del estatuto y de la expansión de las asambleas temáticas, que incorporaron los temas de vivienda, asistencia social, desarrollo económico y social, cultura, deporte y tiempo libre. Hubo un cambio importante en el Consejo al eliminar la representación de las entidades de la sociedad civil, por lo que la representación se redujo desde entonces a las asambleas regionales. Por supuesto, se anuló la visión neocorporativista y se pasó a una visión del PP más cercana a las políticas públicas y, por tanto, donde prevalecía la idea de modernización administrativa y de articulación de la Administración con el PP.

En el ciclo 2003-2004, la división territorial de la ciudad se amplió a 22 regiones. El Consejo pasó a incluir 22 representantes de las asambleas (sociedad civil) y 15 del gobierno. También hubo un cambio organizativo importante: la Coordinación del PP se trasladó del gabinete del alcalde a la Secretaría de gobierno (donde continúa hoy en día). De esta forma, se trató de fomentar la relación del PP con las secretarías municipales, profundizando así la similitud con el modelo de democracia y modernización participativa de Sintomer et al. (2013).

En 2005, se puso en marcha un nuevo período del PP. Los principales cambios fueron que los ciclos se ampliaron de uno a dos años; y los mandatos de los consejeros 
y representantes de los barrios también a dos años. A partir de entonces, el plan de inversiones del municipio fue valorado y aprobado por los consejeros con periodicidad bianual. También hubo una ampliación del número de representantes del Consejo del PP: a partir de entonces son un total de 74 miembros, de los cuales 44 son elegidos por la sociedad civil en asambleas y foros regionales, y 30 son nombrados por el gobierno. Esta es la configuración actual del PP en Guarulhos.

Una característica distintiva del PP de Guarulhos sobre otras experiencias analizadas aquí es la existencia de los Foros Regionales. Aunque también los hay en Diadema, donde se trata de reuniones con los líderes de las entidades más importantes de la sociedad civil, en Guarulhos son espacios en los que potencialmente se reúnen delegados y consejeros de una región acompañados por la Coordinación del PP, y cuyas reuniones se celebran en años impares. Esto permite una mayor sustentación regional de movilizaciones para el PP.

En cuanto a la situación del PP en el gobierno municipal, se puede decir que en Guarulhos existe más proximidad con los órganos administrativos, a diferencia de la estructura de Diadema, más conectada a coordinaciones de contacto con grupos sociales específicos. Como podemos ver en la figura 2, la Coordinación del Presupuesto Participativo depende de un departamento del gobierno y eso supone funciones fuertemente administrativas. La Secretaría de gobierno y, de manera específica el Departamento de Control y Gestión, tiene la función de identificación y autorización (o no) de los gastos, función que generalmente en las alcaldías brasileñas está vinculada a la Secretaría de Finanzas. Por lo tanto, la Coordinación del PP en Guarulhos está más cerca del centro de las decisiones sobre el presupuesto de forma semejante a lo que sucede en Sao Bernardo do Campo, y a diferencia del municipio de Diadema del de Osasco como se verá más adelante.

Figura 2.

GESTIÓN DEL PRESUPUESTO PARTICIPATIVO EN GUARULHOS

GESTIÓN DEL PRESUPUESTO PARTICIPATIVO GUARULHOS

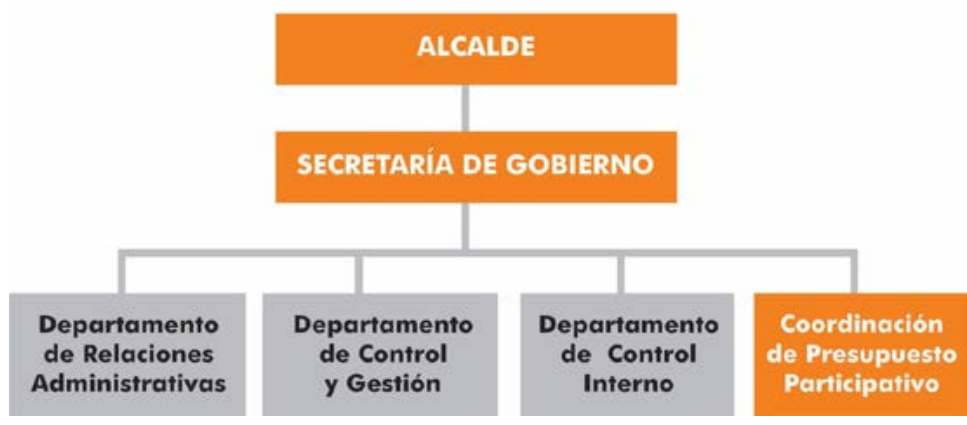

Fuente: elaboración propia basada en la investigación sobre el organigrama de la alcaldía de Guarulhos. 
Con relación al perfil asociativo del Consejo del PP, hay una particularidad en la recogida de datos en Guarulhos, ya que fueron facilitados por el propio equipo del PP del municipio, es decir, siguiendo una metodología diferente a los demás casos. Esto hace que la recopilación de información sobre los consejeros sea sustancialmente menor que en los otros tres casos. Esto, sin embargo, no invalida el uso de la información, ni de los vínculos observados con los partidos políticos, asociaciones de vecinos, organizaciones de la sociedad civil, movimientos sociales y otros consejos.

\section{SOCIOGRAMA 2.}

Guarulhos. Consejo del Presupuesto Participativo. Representantes de la Sociedad CIVIL (2011-2013)

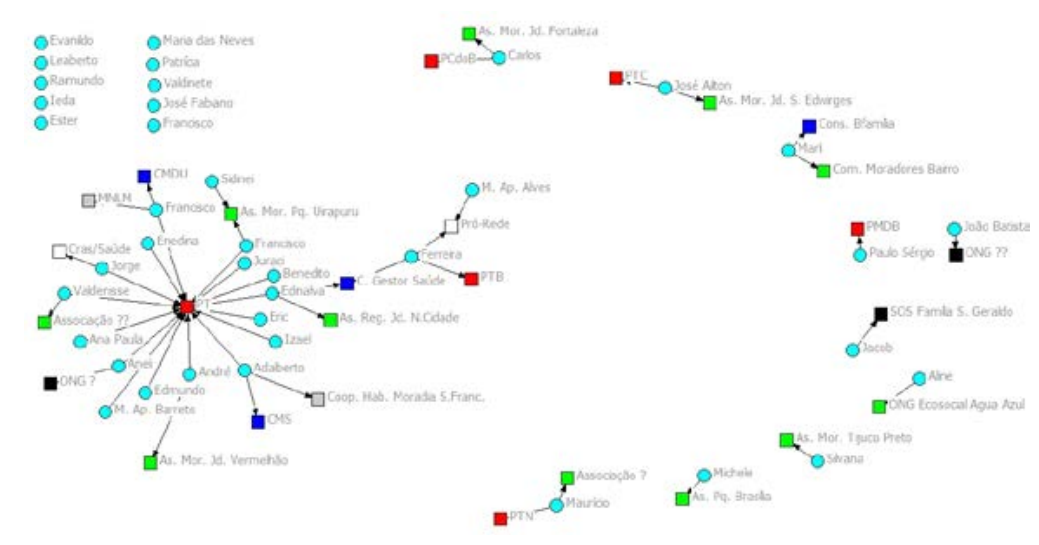

Fuente: elaboración propia basada en las entrevistas.

En el sociograma de Guarulhos, el PT es un nodo central en la red. También existen importantes vínculos entre los consejeros y las asociaciones de vecinos, por lo que la representación territorial se enfatiza en estas asociaciones locales, un factor de agregación y de búsqueda de representación que ciertamente está vinculado a la existencia de Foros Regionales en el PP, de manera que hay cuatro enlaces de representación directa de los consejeros vinculados a las asociaciones de vecinos sin la mediación de los partidos políticos. Hay también un conjunto de diez participantes aparentemente sin vínculos institucionales.

\section{Osasco}

El PP empieza sus actividades en Osasco el primer día de gobierno de Emidio de Souza en 2005. Los encuestados -consejeros o miembros del gobierno- señalan siempre que la primera medida adoptada por el alcalde fue la instalación del PP, como marca distintiva de su gestión. 
En el primer período de gobierno, la coordinación del PP estaba a cargo de un líder histórico del PT de la ciudad. Esto indica el alto nivel de prioridad del PP para dicho gobierno. El primer cambio se produce por la salida de esta persona, al ser elegida presidente municipal del PT en las elecciones internas del partido. La nueva coordinadora, más joven, estuvo en el cargo desde 2007 a 2010 y tenía un perfil de organizadora junto a una posición de «abogada» del PP en el gobierno. A pesar de que había comenzado como una marca de la gestión, el PP pasó a una posición aislada en la alcaldía (como se muestra en la figura 3). En los períodos de cierre de la pieza del presupuesto, la coordinadora del PP intentaba presentar al conjunto de los secretarios las demandas del PP, esperando que se incorporaran en los planes de las secretarías.

El PP se mantuvo mediante las asambleas de consulta, la propuesta de demandas, la elección de los delegados y consejeros del Consejo del Presupuesto Participativo y la capacitación de los consejeros.

Figura 3

Gestión del Presupuesto Participativo - Osasco

\section{GESTIÓN DEL PRESUPUESTO PARTICIPATIVO OSASCO}

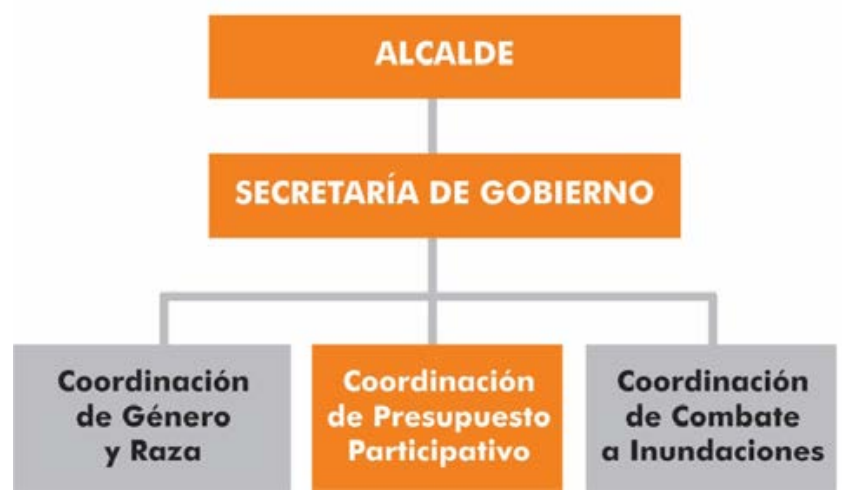

Fuente: Elaboración propia basada en la investigación sobre el organigrama de la Alcaldía de Osasco.

Esta ubicación de la Coordinación del Presupuesto Participativo, en medio de dos Coordinadoras con poca conexión temática entre sí (género y raza, y lucha contra las inundaciones), indica que la estructura de participación estaba lejos de las estructuras de toma de decisiones sobre la configuración del presupuesto. La situación del PP, ya difícil hasta el año 2010, empeora en 2011 cuando, en un cambio del equipo de gobierno, la coordinadora del PP va a ocupar un puesto en la Secretaría de Cultura del municipio. Al principio, no hay ninguna indicación de un nuevo coordinador. Meses más tarde, sin embargo, el ex defensor (ouvidor, en Brasil) del pueblo del municipio comienza a acumular las funciones de Jefe del Gabinete del Alcalde y Coordinador del Presupuesto Participativo. Eso evidencia lo poco que se invierte en el PP, ya que la 
otra función es estratégica para la agenda del alcalde, prioridad política de la administración municipal y una actividad extremadamente absorbente.

La información que he recibido a través de entrevistas con los miembros del equipo y consejeros del PP, en 2012, es que el PP prácticamente fue cerrado, sin dejar de ser una estructura aproximada de una docena de empleados. Esta situación se suma a otra, la creación de un canal alternativo de conexión del alcalde con la "comunidad» en los barrios de la ciudad - el llamado Grupo de Trabajo de Interlocutores (GTI), señalado en las noticias publicadas por el sitio web del gobierno, como «representantes de la administración municipal en las comunidades de los barrios de la ciudad.» ${ }^{3} \mathrm{Se}$ emplearon cerca de 80 personas, incorporadas a la administración municipal después de las elecciones de 2010, encargadas de recorrer las comunidades para anticipar sus demandas y llevar a cabo una relación más estrecha con la población local. La propuesta se había originado directamente desde el núcleo central del gobierno.

En las entrevistas, casi la totalidad de los consejeros señaló la creación del GTI como un elemento importante para la "decadencia» del PP de Osasco. Ya que este conjunto de personas nombradas haría exactamente la actividad de intermediación que los consejeros del PP estaban legitimados para llevar a cabo, al estar elegidos por las asambleas regionales para ser representantes de estas comunidades en sus demandas ante el gobierno municipal. Desde 2011 no se llevaron a cabo elecciones al Consejo, estando formado por aquellos «delegados más activos», los que asistían a las reuniones convocadas por la Coordinación para una rendición de cuentas ocasional e informal de los secretarios del gobierno.

Un elemento importante de análisis se refiere a la diferencia entre el perfil de los consejeros del PP en 2009, en la primera etapa de la investigación, y en 2012, a nuestro regreso a Osasco. Hubo una disminución gradual de los militantes del PT en el PP, lo que se puede explicar en parte por la creación de la GTI. Se produjo una disminución considerable en el estatus político del PP y por ello dejo de ser disputado por los afiliados del PT. El Consejo del PP, a partir de entonces, fue ocupado cada vez más por afiliados de los partidos más pequeños en el contexto político de la ciudad.

Es necesario añadir una información muy importante. Desde su creación, el Consejo del PP de Osasco está, formado únicamente por representantes de la sociedad civil, siendo así el único de este tipo entre los casos estudiados. Este elemento, nos parece que influyó en su vaciamiento más allá de la presencia de los de GTI como competidores de los consejeros.

3. http://www.osasco.sp.gov.br/InternaNot.aspx?id=4680 . Consulta: 14 noviembre 2012. 
Sociograma 3.

Osasco . Consejo del Presupuesto Participativo. Representantes de la Sociedad CiVIL (2010-2012)

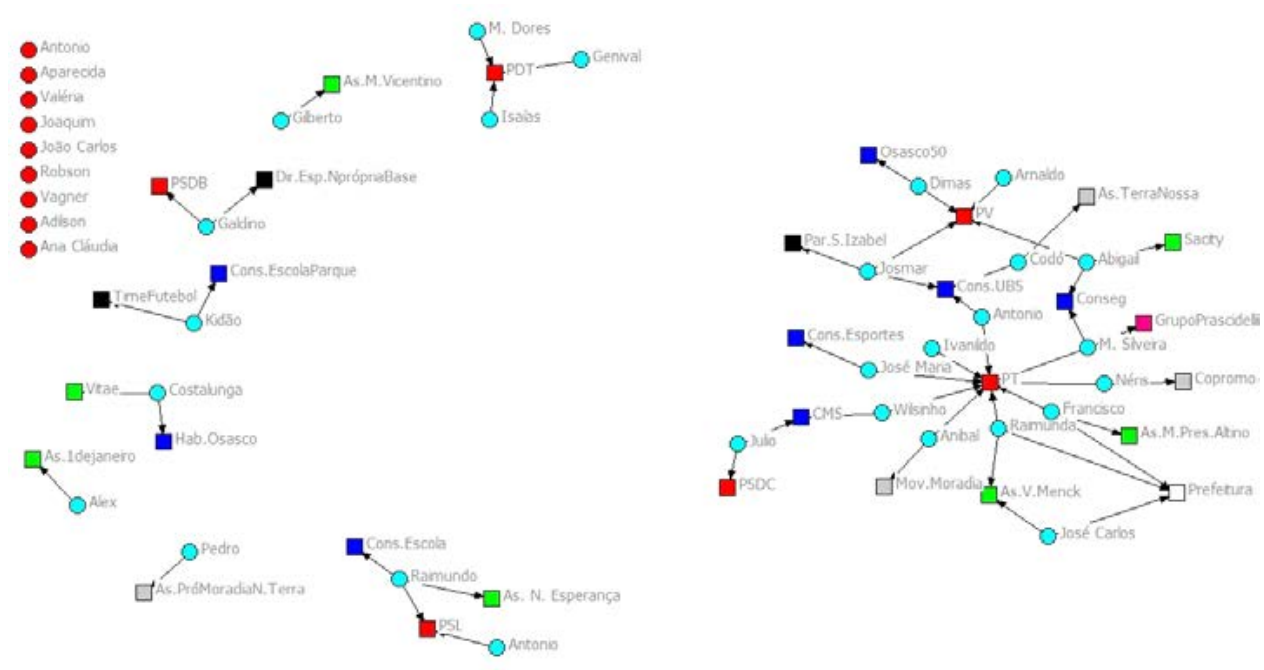

Fuente: elaboración propia basada en las entrevistas.

La presencia solamente de representantes de la sociedad civil corrobora una red de consejeros donde el PT tiene una posición menos central y el PV también es relevante en el conjunto principal. En nuestras investigaciones fuimos informados de que parte de los integrantes del PV eran afiliados del PT y cambiaron al otro partido porque estaban descontentos con los cambios y por la pérdida de poder del PP en la ciudad. Hay muchas conexiones con elementos cercanos al gobierno (cuadrados azules) y algunos ejemplos de conexiones restringidas a las asociaciones de vecinos, como en el caso de Guarulhos. Hay, también, nueve personas (círculos rojos) sin ninguna información de vínculo.

\section{São Bernardo do Campo}

A partir de 2009 empieza la experiencia de PP de São Bernardo do Campo (SBC), en el primer año de mandato de Luiz Marinho (PT) como alcalde. Hay una coyuntura de factores que hace que la administración municipal que se instala en la ciudad tenga un contenido estratégico para el PT en el estado de São Paulo. SBC es la ciudad de Lula, importante simbólicamente para el nacimiento del PT, donde se produjeron las huelgas dirigidas por él a finales de los setenta. Sin embargo, desde hace décadas, SBC ha estado gobernada por partidos de la derecha en el espectro político.

Luiz Marinho es un líder sindical importante, fue presidente de la Central Unitaria de Trabajadores (CUT), entre 2003 y 2005, la central sindical vinculada al PT. 
Después de eso, fue ministro de Trabajo y Empleo, y con posterioridad ocupó la cartera de Seguridad Social en el primer mandato de Lula. Por tanto, la vuelta de Marinho a SBC, para competir en las elecciones a la alcaldía en 2008, se revistió de gran expectativa en la ciudad y entre los más cercanos al grupo político de Lula.

Marinho ganó las elecciones y organizó un gobierno con perfil técnico y político muy experimentado en otras administraciones del PT en la región y el gobierno federal. Es importante tener en cuenta la presencia de Nilza Oliveira, esposa de Luiz Marinho, en la Secretaría de Presupuesto y Planificación Participativa (SPPP). Ella había participado en la administración municipal de Santo André, ciudad que en el segundo período de gobierno de Celso Daniel (PT), de 1997 a 2002, desarrolló una de las experiencias de PP con más éxito de Brasil, modelo para SBC, Guarulhos y muchas otras experiencias.

El estatus del PP en el gobierno de Marinho en SBC es similar al del gobierno de Celso Daniel en Santo André. El PP estaba directamente relacionado con la gestión del presupuesto y la planificación de las acciones del gobierno de la ciudad. Es, en palabras de la secretaria, pensar en la participación como un «método de gobierno", un término que también es utilizado por Gilberto Carvalho y Pedro Pontual (respectivamente ministro jefe y director de Participación Social en la Secretaría General de la Presidencia en el primer gobierno de Dilma Rousseff). Ambos eran miembros claves del gobierno de Celso Daniel en la alcaldía de Santo André.

Figura 4.

Gestión del Presupuesto Participativo. São Bernardo do Campo

GESTIÓN DEL PRESUPUESTO PARTICIPATIVO SÃO BERNARDO DO CAMPO

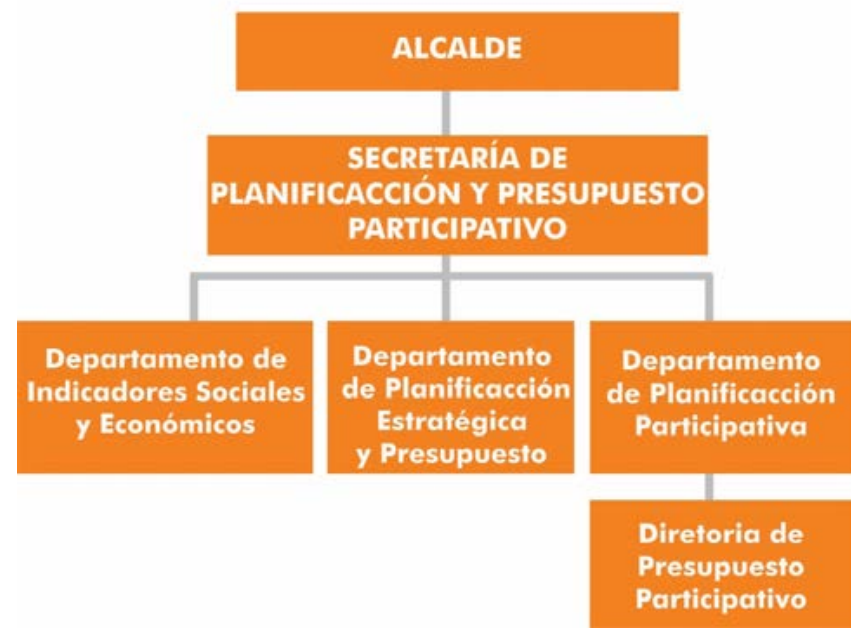

Fuente: elaboración propia a partir del organigrama de la alcaldía de São Bernardo do Campo. 
El proceso de participación social en la elaboración del presupuesto público en SBC comenzó en 2008 con la construcción del programa de gobierno de Marinho en las elecciones. Después de la victoria en las elecciones, la redacción del Plan Plurianual (PPA) se llevó a cabo desde la plataforma electoral de Marinho, expresada en su programa de gobierno. Esta fue una elaboración "participativa", de una manera similar a la realizada en las experiencias del PP, con la celebración de asambleas por distrito del territorio de la ciudad y la elección de los delegados para supervisar la redacción de la propuesta del PPA, hasta su finalización por el gobierno y envío al Legislativo del municipio.

Este proceso, que tuvo lugar en 2009, proporcionó los parámetros por los que al año siguiente comenzó el PP de SBC, bajo el modelo de las asambleas regionales con elección de propuestas y un representante regional para la composición del Consejo del PP. Este, en SBC, se llama Consejo Municipal del Presupuesto (CMP), y decide en todo el proceso de presupuesto de la ciudad y no sólo sobre las demandas de los PP.

Se trata de un consejo paritario con veinte representantes de la sociedad civil -elegidos en las veintes asambleas regionales del PP- y veinte representantes del gobierno municipal, de sus distintas secretarías y autoridades, bajo la coordinación de la SPPP. Se basa en el modelo de Santo André, en especial en lo que respecta a la composición y funciones del CMO. El modelo de consejo paritario $-50 \%$ de los representantes del gobierno y $50 \%$ de representantes de la sociedad civil- fue defendido por Celso Daniel contra Porto Alegre para hacer posible la coexistencia de una doble soberanía: la que proviene de las elecciones y la del proceso de participación en el PP (Pontual y Silva, 2002; Teixeira, 2013).

El caso de SBC es el que nos parece más cercano al modelo que vincula democracia y modernización participativa (Sintomer et al., 2013). Tras una importante reforma en la institucionalización de la elaboración del presupuesto, el proceso participativo culmina con un Consejo paritario que decide sobre la totalidad del presupuesto. No es casualidad, por tanto, que en SBC la presencia del PT como nodo de la red central es aún más pronunciada que en los otros tres casos. El PT articula a casi todos los consejeros, 16. Los cuatro consejeros que no se relacionan con el PT, están afiliados a partidos políticos, exeptuando uno. Así, de dichos partidos dos se oponen al PT (PSDB, PPS) y dos lo apoyan (PTB, PDT) ${ }^{4}$. Esto demuestra que la competencia por un puesto en la CMP está fuertemente ligada a la competencia electoral de los partidos. Al mismo tiempo, hay una diferencia explícita entre los enlaces de algunos asesores a sectores internos del PT, así como a los grupos de apoyo de algunos candidatos, que muestra una interacción bastante consistente con las disputas internas en el PT y en el gobierno. Esto también tiene que ver con la calidad de los datos, es decir, la información transmitida por los consejeros encuestados.

4. PSDB: Partido Social Democracia Brasileña; PPS: Partido Popular Socialista; PTB: Partido Laboral (Trabalhista) Brasileño; PDT: Partido Democrático Laboral (Trabalhista). 
SOCIOGRAMA 4.

São Bernardo do Campo. Consejo Municipal del Presupuesto. Representantes de la SoCIEDAd Civil (2010-2012)

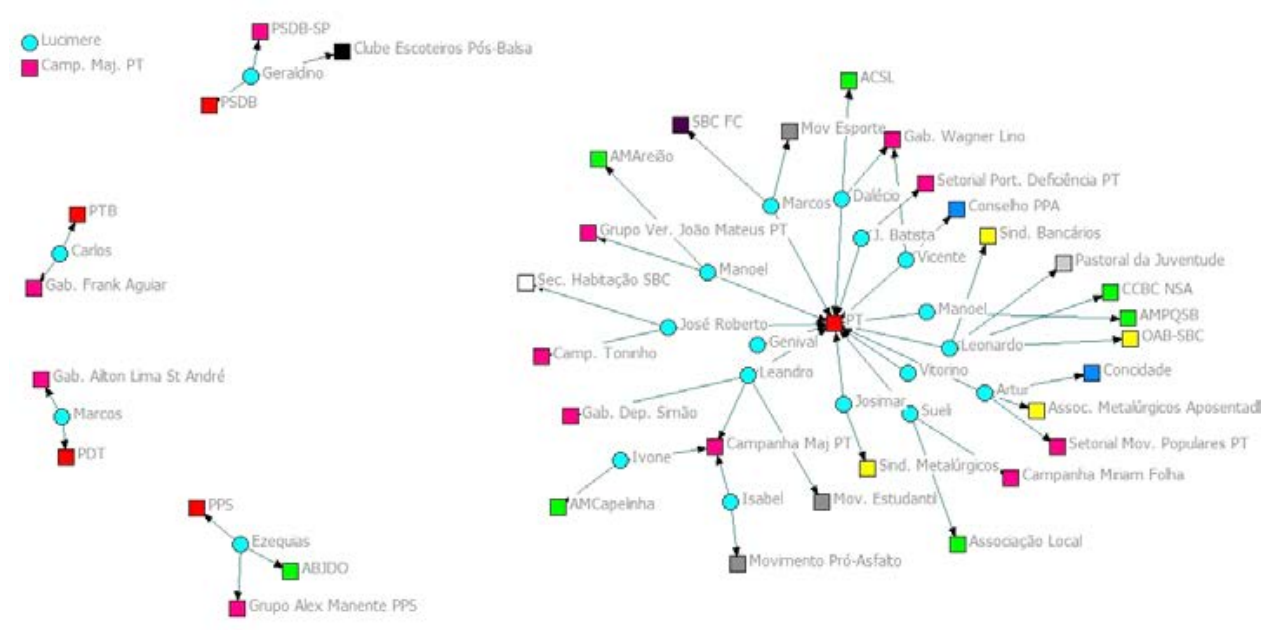

Fuente: elaboración propia basada en las entrevistas.

Sin embargo, también se observan muchos vínculos con los consejeros de las asociaciones de vecinos (7), grupos sindicales (4) y movimientos sociales (4), sobre todo entre los vinculados al PT. No se puede decir lo mismo de los otros cuatro consejeros afiliados a otros partidos políticos, excepto el consejero del PPS.

Es de destacar que apareció muy poco en SBC el vínculo de los consejeros del PP con otros consejos de políticas públicas -solo en dos casos-, de modo que parece que hay una «especialización» de los consejeros en el PP, quizás por la fuerte efectividad del $\mathrm{PP}$ en el contexto del gobierno, que hace que el CMP sea un espacio de disputa fuerte.

En comparación con los consejos de otras ciudades, se puede ver que la red SBC tiene más densidad. Esto se debe a la intensidad del PT como nodo central de la red y puede indicar que en ese caso el vínculo partidario (con el PT) y territorial con las asociaciones de vecinos es más significativo que en Diadema y Guarulhos.

\section{CONCLUSIONES}

En este artículo se ha llevado a cabo una comparación entre cuatro experiencias de PP (Diadema, Guarulhos, Osasco y São Bernardo do Campo), con el fin de comprobar cómo las diferentes formas de organización del PP -especialmente su ubicación en la estructura administrativa de la alcaldía y la forma de sus canales de mediación entre la sociedad y el gobierno- generan distintos perfiles institucionales de la sociedad civil movilizada. 
El análisis de los casos indica que existe una considerable variación en la ubicación de la estructura coordinadora del PP en la administración municipal. Hay por lo menos tres opciones de ubicación: a) incrustados o muy cerca de la estructura institucional de planificación y decisión sobre el presupuesto (São Bernardo do Campo y, en menor medida, Guarulhos); b) conectado a la estructura de diálogo con grupos específicos, como en Diadema, y c) a distancia de la estructura de planificación y sin vínculos perceptibles con otras estructuras para el diálogo con la población, como en Osasco. La tendencia observada es que un mayor estatus político en el contexto de la Administración Municipal se relaciona con la cercanía de la estructura participativa con el marco de planificación y decisión sobre el presupuesto. En cambio, una desconexión grande con estas estructuras, como en el caso de Diadema y, más enfáticamente, Osasco, indica un estatus político inferior del PP.

En los casos estudiados, el estatus político más elevado de la experiencia de São Bernardo do Campo se ve reforzado por las características asumidas por el Consejo conectado al PP, el Consejo Municipal del Presupuesto, instancia paritaria (50 \% del gobierno y $50 \%$ de representantes de la sociedad civil provenientes del proceso del PP) que delibera sobre la propuesta presupuestaria que se enviará a la legislatura. Aportar representantes de la sociedad civil para la discusión general del presupuesto de la ciudad es un indicador de alto estatus político del proceso participativo en el municipio.

En los casos de Guarulhos y Diadema encontramos estructuras intermedias en las que la sociedad civil tiene representación mayoritaria en los consejos. Y Osasco es el caso de estatus político más bajo de entre los estudiados, donde sólo hay representantes de la sociedad civil en un Consejo que se reúne con poca frecuencia o ha dejado de hacerlo. Por tanto, considerando el análisis de los casos, hay más posibilidades de que el consejo sea efectivo, es decir, genere decisiones políticas reales sobre el presupuesto, cuanta más presencia compartida exista entre los funcionarios del gobierno y los miembros de la sociedad civil. Cuando esto no ocurre, la tendencia es que el consejo sea poco efectivo y la participación caiga en descrédito.

Finalmente, la combinación de estas dos características -mayor estatus político e igualdad de representación entre el gobierno y la sociedad civil en el consejo del PPgenera la tendencia de que el perfil político e institucional de los consejeros de la sociedad civil esté más cerca de la organización de los partidos políticos en la ciudad y de la coalición gobernante en el poder. Esto explica la conexión más directa entre el Consejo de São Bernardo do Campo y la afiliación de consejeros al PT y, por otro lado, la conexión más débil entre el PP de Osasco y el partido gobernante. En São Bernardo do Campo hay una presencia muy fuerte del PT entre los consejeros y es también donde los sindicatos y las organizaciones profesionales aparecen con más fuerza; ello puede deberse a las características del PT en la ciudad y al origen sindical del alcalde. En cambio, en Osasco hay más fragmentación en la afiliación partidaria y también mayor presencia de personas vinculadas directamente al gobierno o a otro tipo de consejo, que puede denotar el desinterés de la sociedad civil local, incluido el más relacionado con el PT, en hacerse presente en el Consejo del PP. 
En estos casos, la tipología propuesta por Sintomer et al., (2013), y adoptada en este artículo con algunos cambios, nos lleva a caracterizar a São Bernardo do Campo como el caso que se adapta mejor a la articulación entre el modelo de democracia participativa y el modelo de modernización participativa. La estructura participativa está centralizada en la Secretaria de Planeación del gobierno y hay paridad en el Consejo. El síntoma de que el Consejo funciona bien es la fuerte presencia de los partidos políticos en el mismo; es decir, la parte más organizada de la sociedad se disputa los puestos en la cumbre del poder compartido.

Guarulhos presenta una situación donde hay aspectos propios de la modernización participativa - una presencia fuerte cerca del núcleo central del gobierno-. En un primer momento estuvo cerca del neocorporativismo, pero después fue desplazándose hacia una democracia de proximidad, con una relación menos fuerte con las entidades de la sociedad civil y más de escucha de una parte de la sociedad relacionada con las asociaciones comunitarias.

Diadema presenta una división clara entre una democracia de proximidad interna en las estructuras ordinarias del PP y un neocorporativismo en los foros regionales. Existe una preocupación fuerte por identificar nuevos actores que puedan acercarse al PP y esto lleva a los gestores a intentar siempre nuevas formas de movilización sociopolítica.

Por último, Osasco inició la experiencia de PP con una conexión muy clara con la figura del alcalde, pero al cabo de pocos años cambió hacia una versión rebajada del $\mathrm{PP}$, donde la democracia de proximidad se establece con poco poder de toma de decisiones de los consejeros, lo que se percibe en la menor disputa por los puestos en el Consejo del PP.

En este artículo se ha demostrado cómo las experiencias del PP que tienen una mayor centralidad en la Administración Municipal, es decir, un mayor estatus político, generan una situación por la cual el perfil asociativo de sus consejeros de la sociedad civil está políticamente más cerca de dicha Administración. Además, es posible considerar a partir del análisis de los casos que cuanto mayor sea el estatus político, mayor será el espacio de la toma de decisiones en el contexto del proceso participativo -el consejo del PP, que será disputado por representantes con conexiones más fuertes con los partidos políticos-. Tenemos, por lo tanto, una dinámica de conflicto por los recursos políticos, donde los grupos más organizados dirigen hacia el PP sus estrategias de ocupación del espacio político, con la obtención de ventajas comparativas sobre los menos organizados, similar a lo que ocurre en el campo de la democracia representativa.

Es posible que en otros casos fuera de Brasil encontremos diferencias en este sentido, y somos conscientes de que esta es una importante limitación del artículo. Pero creemos que es imprescindible hacer una evaluación de cómo el sistema partidario y electoral se comporta en relación con las oportunidades de participación en el PP. El sistema brasileño es multipartidista y fragmentado y todas las posibilidades de participación de la sociedad civil están fuertemente disputadas por afiliados a los partidos, que buscan posicionarse en mejores condiciones para aprovechar las aperturas de espacio en la política, y especialmente para influir a los gobiernos. 


\section{Referencias}

Avritzer, Leonardo. 2003. «O orçamento participativo e a teoria democrática: um balanço crítico», en Leonardo Avritzer y Zander Navarro (eds.), A inovação democrática no Brasil: o orçamento participativo. São Paulo: Cortez.

Avritzer, Leonardo. 2009. Participatory institutions in democratic Brazil. Baltimore: Johns Hopkins University Press.

Baiocchi, Gianpaolo. 2005. Militants and citizens: the politics of participatory democracy in Porto Alegre. Stanford, California: Stanford University Press.

Baiocchi, Gianpaolo y Patrick Heller. 2009. «Representation by design? Variations on participatory reforms in Brazilian municípios», en Olle Törnquist, Neil Webster y Kristian Stokke (eds.), Rethinking popular representation. New York: Palgrave Macmillan.

Baiocchi, Gianpaolo, Patrick Heller y Marcelo Kunrath. 2011. Bootstrapping democracy: transforming local governance and civil society in Brazil. Stanford, California: Stanford University Press.

Filomena, Cesar. 2006. O agonismo das relaçôes sociais do partido, dos espaços públicos da sociedade civil e do sistema administrativo estatal: a experiência da Administração Popular em Porto Alegre. Maestria. Programa de Pós-Grado en Ciências Sociales, PUC-RS, Porto Alegre, Brasil.

Ganuza, Ernesto y Baiocchi, Gianpaolo. 2013. «Para além da linha: o orçamento participativo como instrumento», en Nelson Dias (ed.), Esperança democrática: 25 anos de orçamentos participativos no mundo. São Brás de Alportel: Associação In Loco.

Leubolt, Bernhard, Wagner Romão, Joachim Becker y Andreas Novy. 2012. «Re-evaluating participatory governance in Brazil», en Barry Cannon y Peadar Kirby (eds.), Civil society and the state in left-led Latin America: challenges and limitations to democratization. New York: Zed Books.

Lüchmann, Ligia. 2014. "25 anos de Orçamento Participativo: algumas reflexões analíticas», Política \& Sociedade, 13 (28): 167-197.

Hanneman, Robert. 2001. Introduction to social network methods. Riverside: University of California.

Mische, Ann. 2009. Partisan publics: communication and contention across Brazilian youth activist networks. Princeton, New Jersey: Princeton University Press.

Montambeault, Françoise. 2012. ¿Aprendiendo a ser «mejores demócratas»? El papel de las prácticas informales en las experiencias de presupuestos participativos en Brasil, en Maxwell Cameron, Eric Hershberg y Kenneth E. Sharpe (eds.), Nuevas instituciones de democracia participativa en América Latina: la voz y sus consecuencias. México: FLACSO México.

Navarro, Zander. 2003. «O 'Orçamento Participativo' de Porto Alegre (1989-2002): um conciso comentário crítico», en Leonardo Avritzer y Zander Navarro (eds.), $A$ inovação democrática no Brasil: o orçamento participativo. São Paulo: Cortez. 
Nylen, Willian. 2002. "Testing the empowerment thesis: the participatory budget in Belo Horizonte and Betim, Brazil», Comparative Politics, 34, 127-145.

Pontual, Pedro y Carla Silva. 2002. «Participação popular nos governos petistas: trajetórias, mecanismos e caráter», en Inês Magalhães et. al.(eds.), Governo e cidadania: balanço e perspectivas do modo petista de governar. São Paulo: Fundação Perseu Abramo.

Porto de Oliveira, Osmany. 2016. Embaixadores da participação: a difusão internacional do orçamento participativo a partir do Brasil. São Paulo: Annablume.

Romão, Wagner. 2011. "Conselheiros do orçamento participativo nas franjas da sociedade política», Revista Lua Nova, 84: 219-244.

Santos, Boaventura. 1998. «Participatory budgeting in Porto Alegre: toward a redistributive democracy», Politics \& Society, 26 (4): 461-489.

Sintomer, Yves y Ernesto Ganuza. 2011. Democracia participativa y modernización de los servicios públicos: investigación sobre las experiencias de presupuesto participativo en Europa. Transnational Institute.

Sintomer, Yves, Carsten Herzberg y Anja Röcke. 2013. «Modelos transnacionais de participação cidadã: o caso do orçamento participativo», en Nelson Dias (ed.), Esperança democrática: 25 anos de orçamentos participativos no mundo. São Brás de Alportel: Associação In Loco.

Teixeira, Ana Cláudia. 2003. «O OP em pequenos municípios rurais: contextos, condiçōes de implementação e formatos de experiência», en Leonardo Avritzer y Zander Navarro (eds.), A inovação democrática no Brasil: o orçamento participativo. São Paulo: Cortez.

Teixeira, Ana Cláudia. 2013. Para além do voto: uma narrativa sobre a democracia participativa no Brasil (1975-2010). Tatagiba, Luciana (dir.), Universidade Estadual de Campinas.

Wampler, Brian. 2004. "Instituições, associações e interesses no orçamento participativo de São Paulo», en Leonardo Avritzer (ed.), A participação em São Paulo. São Paulo: Editora Unesp.

Wampler, Brian. 2007. Participatory budgeting in Brazil: contestation, cooperation, and accountability. University Park: The Pennsylvania State University Press. 
Presentado para evaluación: 2 de junio de 2016.

Aceptado para publicación: 26 de octubre de 2016.

\section{WAGNER ROMÃO}

wromao@unicamp.br

Es doctor en Sociología por la Universidad de São Paulo. Profesor del Departamento de Ciencia Política de la Universidad Estatal de Campinas. Investigador del Núcleo de Estudios sobre Movimientos Sociales, Participación y Acción Colectiva (NEPAC-UNICAMP) y del Núcleo Democracia y Acción Colectiva en el Centro Brasileño de Análisis y Planeamiento (NDAC-CEBRAP). Sus líneas de investigación versan sobre las relaciones entre participación, democracia y políticas públicas. 